\title{
Impact of a Mindfulness Based Stress Reduction Intervention on the Emotional Intelligence, Burnout and Anticipated Turnover Among Critical Care Nurses
}

\author{
Carmen Corder*, Jeffrey N Doucette and Nancy L Sweeney \\ Department of Nursing, University of Tennessee Martin, USA
}

*Corresponding author: Carmen Corder, Department of Nursing, University of

Tennessee Martin, USA.

\section{Abstract}

Background: Burnout and turnover is a significant problem within critical care. Due to the various outcomes of burnout and turnover, including their impact on the quality of patient care, it is imperative to consider strategies to reduce burnout and turnover of critical care nurses.

Research objective: To determine the effectiveness of a mindfulness-based stress reduction intervention on the emotional intelligence, burnout inventory scores, and anticipated turnover among a group of critical care nurses.

Research design: This study used a pre-experimental, pre- and post-test design. Participants and research context: A total of 32 intensive care nurses were selected from a large tertiary care facility in West TN. Data collection instruments were a "demographics questionnaire", "Genos Concise Emotional Intelligence Inventory", Maslach Burnout Inventory[1], and the "Anticipated Turnover Scale".

Ethical Considerations: Informed consent from participants and research approval was granted by the Old Dominion University Institutional Review Board.

Findings: Findings showed that nurses had moderate levels of burnout an anticipated turnover. Results revealed that participation in the mindfulness-based stress reduction intervention was associated with an increase in emotional intelligence and decreases in both burnout and anticipated turnover.

Discussion: Many findings in this study are consistent with those of other, similar studies.

Conclusions: An overall decrease in post-intervention burnout and anticipated turnover substantiates the use of mindfulness as a useful retention strategy.

Keywords: Mindfulness; Emotional intelligence; Burnout; Turnover

\section{Introduction}

Turnover of experienced nurses in intensive care is a critical problem that can be attributed to a disproportionate amount of stress and burnout among this specialized group of RNs [2]. Studies indicate that an interrelated combination of work-related and personal factors influence stress levels and decisions to leave the ICU; however, high levels of EI have been shown to protect against work-related stressors. Nurses with high levels of EI possess superior clinical performance and improved overall well-being [3].

\section{Objectives}

- To determine the impact of participation in a MBSR on the EI of critical care nurses.

- To determine the impact of participation in a MBSR intervention on the burnout inventory scores of critical care nurses. 
- To determine the impact of participation in a MBSR intervention on the anticipated turnover of critical care nurses.

\section{Prevalence of burnout and turnover among critical care nurses}

1. $33 \%$ of critical care nurses display symptoms of severe burnout [4].

2. Annual turnover is estimated to be $25 \%$ to $60 \%$ [5]. Nearly $17.5 \%$ of all newly licensed registered nurses (RN) leave their first nursing job within one year, and that as many as 33.5\% leave within two years (Robert Wood Johnson Foundation [RWJF], 2014).

\section{Consequences of burnout and turnover among critical care nurses}

1. Exhaustion, depersonalization, and/or reduced personal accomplishment within critical care nurses.

2. Higher 30-day mortality rates as being very closely associated with nurse burnout [6].

3. High nurse turnover leads to higher nurse to patient ratios, which results in more frequent occurrences of preventable complications such as pneumonia, falls, decubitus ulcers, and reduced overall patient satisfaction [7].

4. Patient mortality is proven to be significantly higher in ICUs with the highest nurse to patient ratios [8].

Table 1: Demographic data of study participants $(\mathrm{N}=32)$.

\begin{tabular}{|c|c|c|}
\hline Characteristics & Measurement & N=32 \\
\hline \multirow{2}{*}{ Gender } & Female & $12 \%$ \\
\hline Age in years & Male & 35.45 \\
\cline { 2 - 3 } & Age in years & 8.1 \\
\hline Number of years of nursing experience & Years & $53.10 \%$ \\
\hline \multirow{2}{*}{ Highest Level of Nursing Education } & ADN & $46.90 \%$ \\
\hline \multirow{2}{*}{ Highest Level of Non-Nursing Education } & BSN & $28.60 \%$ \\
\cline { 2 - 3 } & Diploma & $42.90 \%$ \\
\cline { 2 - 3 } & Associate & $23.80 \%$ \\
\cline { 2 - 3 } & Bachelor & $4.80 \%$ \\
\hline \multirow{2}{*}{ Religious Preference } & Master & $96.90 \%$ \\
\hline
\end{tabular}

\section{Results}

Table 2: Emotional Intelligence data of participants $(\mathrm{N}=32)$.

\begin{tabular}{|l|c|c|}
\hline \multirow{2}{*}{ Dependent Variable } & Pre-Intervention N=32 & Post-Intervention N= 32 \\
\hline \multirow{3}{*}{ Emotional Intelligence } & Mean: 114.53 & Mean: 122.437 \\
\cline { 2 - 3 } & SD: 13.24 & SD: 14.107 \\
\cline { 2 - 3 } & Range: 51.00 & Range: 60.00 \\
\cline { 2 - 3 } & Minimum: 91.00 & Minimum: 94.00 \\
\cline { 2 - 3 } & Maximum: 142.00 & Maximum: 154.00 \\
\hline
\end{tabular}

\section{Benefits of High Levels of Emotional Intelligence (EI) among Critical Care Nurses}

1. High levels of EI have been shown to protect against workrelated stressors.

2. Nurses with high levels of EI possess superior clinical performance and improved overall well-being [8].

\section{Methods/Intervention}

This research is a quantitative, pre-experimental study. Three dependent variables (EI, burnout, anticipated turnover) were measured using the Geno's Emotional Intelligence Inventory, the Maslach Burnout Inventory, and the Anticipated Turnover Scale, respectively. The pre-intervention questionnaire survey was administered to participants between September 2017- November 2017. Participants completed the researcher led MBSR intervention by February 2018 and were administered a post-intervention survey questionnaire. Data was analyzed for differences in pre and post-intervention scores.

\section{The Study}

The purpose of this research was to evaluate the effect of a mindfulness-based stress reduction intervention on the EI, burnout inventory scores, and anticipated turnover among critical care nurses in a large hospital located in Tennessee (Table 1). 
Following completion of the MBSR intervention, the mean EI score increased from 114.53 to 122.437 (Table 2).

Table 3: Burnout data of participants $(\mathrm{N}=32)$.
Post-intervention data revealed an eight-point increase in the mean burnout inventory score (Table 3 ).

\begin{tabular}{|c|c|c|}
\hline \multirow{2}{*}{ Dependent Variable } & Pre-Intervention N=32 & Post-Intervention N=32 \\
\hline \multirow{3}{*}{ Burnout } & Mean: 56.531 & Mean: 48.562 \\
\cline { 2 - 3 } & SD: 10.993 & SD: 9.986 \\
\cline { 2 - 3 } & Range: 48.00 & Range: 38.00 \\
\cline { 2 - 3 } & Minimum: 35.00 & Minimum: 32.00 \\
\cline { 2 - 3 } & Maximum: 83.00 & Maximum: 70.00 \\
\hline
\end{tabular}

Table 4: Anticipated turnover data of participants $(N=32)$.

\begin{tabular}{|c|c|c|}
\hline \multirow{2}{*}{ Dependent Variable } & Pre-Intervention N=32 & Post-Intervention N= 32 \\
\hline \multirow{3}{*}{ Anticipated Turnover } & Mean: 35.875 & Mean: 32.593 \\
\cline { 2 - 3 } & SD: 11.550 & SD: 10.733 \\
\cline { 2 - 3 } & Range: 39.00 & Range: 40.00 \\
\cline { 2 - 3 } & Minimum: 17.00 & Minimum: 17.00 \\
\cline { 2 - 3 } & Maximum: 56.00 & Maximum: 57.00 \\
\hline
\end{tabular}

Results showed an increase in the mean anticipated turnover score after completion of the MBSR intervention from 32.593 to 35.875 (Table 4).

\section{Conclusion}

Participation in the MBSR intervention resulted in an overall $7 \%$ increase in EI. These findings are consistent with other similar studies. EI is well recognized as an important component in overall staff welfare and the patient care delivered by professional nurses, and therefore must be considered as an important factor when addressing nurse retention [9]. A 14\% decrease in overall mean burnout inventory scores was noted following completion of the MBSR intervention. Prior studies have shown that burnout among critical care health care professionals is associated with the development of post-traumatic stress disorder, alcohol abuse, and even suicidal ideations [10]. It is therefore imperative to develop strategies to diminish burnout and this study has shown mindfulness to be an effective burnout reduction strategy.

A 9\% decrease in overall anticipated turnover intention was noted following completion of the MBSR intervention. Studies have shown a positive correlation between anticipated turnover and actual turnover [11]. Taking this into consideration it is imperative for nurse leaders to implement strategies that will reduce the anticipated turnover of ICU nurses. Hospital administrators, nurse managers and leaders are urged to work towards creating favorable work environments supporting nursing practice and ultimately leading to improved quality of care. Burnout among nurses is associated with reports of poor quality of care, increased patient falls, medication errors, and higher rates of hospitalacquired infections [12]. The introduction of mindfulness could be easily incorporated into new nurse orientation programs and the techniques could be reinforced in continuing education offerings.
Since many of the nurses inquired about receiving something in return for participation, administration could offer incentives such as "pull passes" to facilitate application of mindfulness to reduce burnout, turnover, and improve the emotional intelligence of experienced and new nurses.

\section{Acknowledgement}

None.

\section{Conflict of Interest}

No conflict of interes

\section{References}

1. Maslach C, Jackson S (1981) Maslach Burnout Inventory Human Services Survey.

2. Shoorideh F, Ashktorab T, Yaghmei F, Mahd H (2015) Relationship between ICU nurses' moral distress with burnout and anticipated turnover. Nurs Ethics 22(1): 64-76.

3. Hall L, Johnson J, Watt I, Tsipa A, O Connor DB (2016) Healthcare staff wellbeing, burnout, and patient safety: A systematic review. PLoS ONE 11(7): e0159015.

4. Stempniak M (2016) Burnout common among ICU nurses: 5 tips for retaining new nurses. American Hospital Association.

5. Mealer M, Conrad D, Evans J, Jooste K, Solyntjes J, et al. (2014) Feasibility and acceptability of a resilience training program for intensive care unit nurses. American Journal of Critical Care 23(6): 97-105.

6. Halbesleben J, Rathert C, William E (2013) Emotional exhaustion and medication administration workarounds: The moderating role of nursing satisfaction with medication administration. Health Care Management 38(2): 95-104.

7. Divitia J (2014) Burnout in the ICU: Playing with fire. Journal of Critical Care Medicine 18(3): 127-128.

8. Falk A, Wallin E (2016) Quality of patient care in the critical care unit in relation to nurse: patient ratio: A descriptive study. Intensive and Critical Care Nursing 35: 74-79. 
9. McQueen A (2004) Emotional intelligence in nursing work. Journal of Advanced Nursing 47(1): 101-108.

10. Myhren H, Oivind E, Stokland O (2013) Job satisfaction and burnout among intensive care nurses and physicians. Critical Care Research and Practice.
11. Eberhardt B, Poonam A, Moser S (1995) Moderators of the relationship between job satisfaction and nurses' intention to quit. International Journal of Organizational Analysis 3(4): 394-406.

12. Nantsupawat A, Kunaviktikul W, Turale S, Poghosyan L (2016) Nurse Burnout, Nurse-Reported Quality of Care, and Patient Outcomes in Thai Hospitals. Journal of Nursing Scholarship 48(1): 83-90. 